\title{
CARTOGRAMA SÓCIO-DEMOGRÁFICO COMO SUBSÍDIO PARA ALTERNATIVAS DE DESENVOLVIMENTO: O CASO DO EIXO NORTE DA RMBH
}

\author{
Paulo Eduardo Alves Borges da Silva* \\ Ricardo Alexandrino Garcia \\ Ralfo Edmundo da Silva Matos` \\ Brenner Henrique Maia Rodrigues
}

\section{Resumo}

A Região Metropolitana de Belo Horizonte, igualmente a outras agremiações urbanas do país, apresentou profundas transformações relacionadas a um intenso dinamismo demográfico nas últimas duas décadas. O incremento populacional nas porções periféricas desta região advém, majoritariamente, da busca por melhores condições de vida e oportunidades de emprego. A recente instalação de Grandes Equipamentos no Eixo Norte da região, estimula ainda mais o surgimento de estabelecimentos industriais, comerciais e habitacionais. Estas alterações podem trazer inúmeros enclaves econômicos e ambientais, sendo alvo de investigação de planejadores e de políticas públicas no Estado. O cerne deste artigo é apontar como o mapeamento de diferentes aspectos sócio demográficos possibilitou a formulação de alternativas voltadas a um desenvolvimento equilibrado diante dos impactos previstos para estes municípios ainda carentes de infraestrutura. A base sócia demográfica que compõe a espacialização das informações corresponde à uma área de estudo especial, definida pelos municípios de Sabará, Ribeirão das Neves, Vespasiano, Confins, Lagoa Santa, São José da Lapa, Santa Luzia e norte do município de Belo Horizonte. Assim, do ponto de vista do contexto político administrativo, a área de abrangência do Cartograma contempla total ou parcialmente os oito municípios, cuja unidade geográfica mínima trabalhada foi o setor censitário. Para as estimativas de demanda no período 2005 - 2020, projeção populacional e métodos de áreas mínimas de comparação, foram realizados procedimentos não triviais, que envolveram um complexo banco de dados e operações espaciais com as bases cartográficas de setores urbanos e rurais disponibilizadas pelo IBGE (2000). O conjunto de mapas elaborado permitiu a análise conjunta da situação sóciodemográfica local e o apontamento de alternativas que buscam um desenvolvimento equilibrado para a área, diante das novas e futuras configurações espaciais ali inseridas.

Palavras-chave:Cartograma; Sócio demográfico; RMBH; Eixo Norte; Alternativas.

\section{Abstract}

The metropolitan area of Belo Horizonte, also to other urban associations in the country, had profound transformations related to intense demographic dynamism in the last two decades. The increase in population in the peripheral portions of this region stems, mainly, the quest for better living conditions and employment opportunities. The recent installation of Big Equipments in the North Axis region, further stimulate the emergence of industrial, commercial and residential use. These changes can bring many economic and environmental enclaves, and targeted research and policy planners in the State. The core of this paper is to highlight how the mapping of various socio-demographic factors enabled thedevelopment of alternatives aimed at balanced development in the face of expected impacts for these municipalities still lack infrastructure. The basic socio-demographic component of the spatial

\footnotetext{
*Mestrando em Análise e Modelagem de Sistemas Ambientais/UFMG; pauloeduardoborges@gmail.com

•Dr. Prof. Departamento de Geografia/UFMG; alexandrinogarcia@gmail.com

`Dr. Prof. Departamento de Geografia/UFMG; ralfomatos@yahoo.com.br

`Mestrando em Geografia e Análise Ambiental/UFMG; bhmrodrigues@yahoo.com.br
} 
information corresponds to a particular study area, defined by the municipality of Sabará, Ribeirão das Neves, Vespasiano, Confins, Lagoa Santa, São José da Lapa, Santa Luzia and north of Belo Horizonte. Thus, from the standpoint of political and administrative context, the coverage area of the Cartogram includes all or part of eight counties, whose unit was the minimum geographical worked census tract. Demand estimates for the period 2005 - 2020, population projection methods and minimum areas of comparison procedures were performed non-trivial, involving a complex database and space operations with the cartographic base of the urban and rural areas provided by IBGE (2000). The set of maps prepared allowed the joint analysis of the sociodemographic location and identification of alternatives that seek a balanced development for the area in front of new and future spatial configurations there inserted.

Key words: Cartogram; Socio-demographic; RMBH, Development; Alternatives.

\section{1- INTRODUÇÃO}

Este trabalho está inserido em um estudo de maior grandeza realizado entre novembro de 2007 e maio de 2008 por uma equipe multidisciplinar formada por professores, pesquisadores e estudantes de pós-graduação e graduação do Instituto de Geociências da Universidade Federal de Minas Gerais e do Instituto de Pesquisa e Planejamento Urbano e Regional da Universidade Federal do Rio de Janeiro. A pesquisa denominada Estudo sobre os impactos oriundos de iniciativas localizadas no eixo norte da Região Metropolitana de Belo Horizonte e definição de alternativas de desenvolvimento econômico, urbano e social para o Município de Belo Horizonte incorpora as importantes contribuições - de técnicos e gestores da Prefeitura de Belo Horizonte e outros convidados - oriundas dos encontros de discussão ocorridos ao longo do desenvolvimento do estudo.

Para a realização do seu propósito central - identificar impactos gerados por grandes empreendimentos e projetos no eixo norte de expansão da Região Metropolitana de Belo Horizonte e indicar linhas de atuação em subsídio ao planejamento municipal -, o estudo se desenvolveu segundo três grandes linhas de trabalho: a análise de seis grandes projetos definidos e sua relação com a dinâmica e o desenvolvimento do Município e da Região Metropolitana de Belo Horizonte; a indicação de eixos de atuação do poder público que orientem o planejamento municipal de Belo Horizonte e a realização de um macro diagnóstico econômico, urbano e social da evolução recente da região.

Desse modo, o foco deste artigo - e inerente aos interesses da Cartografia - é apresentar, através de parte da metodologia e das análises sócio demográficas, como a coletânea de mapas contribuiu para a elaboração de alternativas para a região. Para tal é descrita uma breve caracterização da área; parte da metodologia empregada; um recorte das análises sócio demográficas; as considerações sobre o aparato das cartas estabelecidas e a distinção de algumas alternativas políticas sugeridas decorrentes do estudo. 


\section{2 - O EIXO NORTE DA RMBH}

Nos últimos anos, a RMBH tem sido palco de uma série de transformações e estímulos com impactos significativos nas dinâmicas demográficas, socioeconômicas e espaciais do espaço metropolitano. As principais intervenções implantadas, ou que ainda estão em fase de implantação e planejamento, selocalizam nos municípios que compõem o Vetor Norteda RMBH, com destaque para a realocação dos voos para o aeroporto de Confins, a criação do AeroportoIndustrial, a Linha Verde, a construção da Cidade Administrativa e do Rodoanel.

De acordo com o Decreto 44.500 de 03 de Abril de 2007, “o Vetor Norte da RMBH é formado pelos municípios de Ribeirão das Neves, Santa Luzia, Vespasiano, Lagoa Santa, Pedro Leopoldo, São José da Lapa, Confins, Jaboticatubas e pelos bairros localizados na área de influência das administrações regionais de Venda Nova e Norte do município de Belo Horizonte".

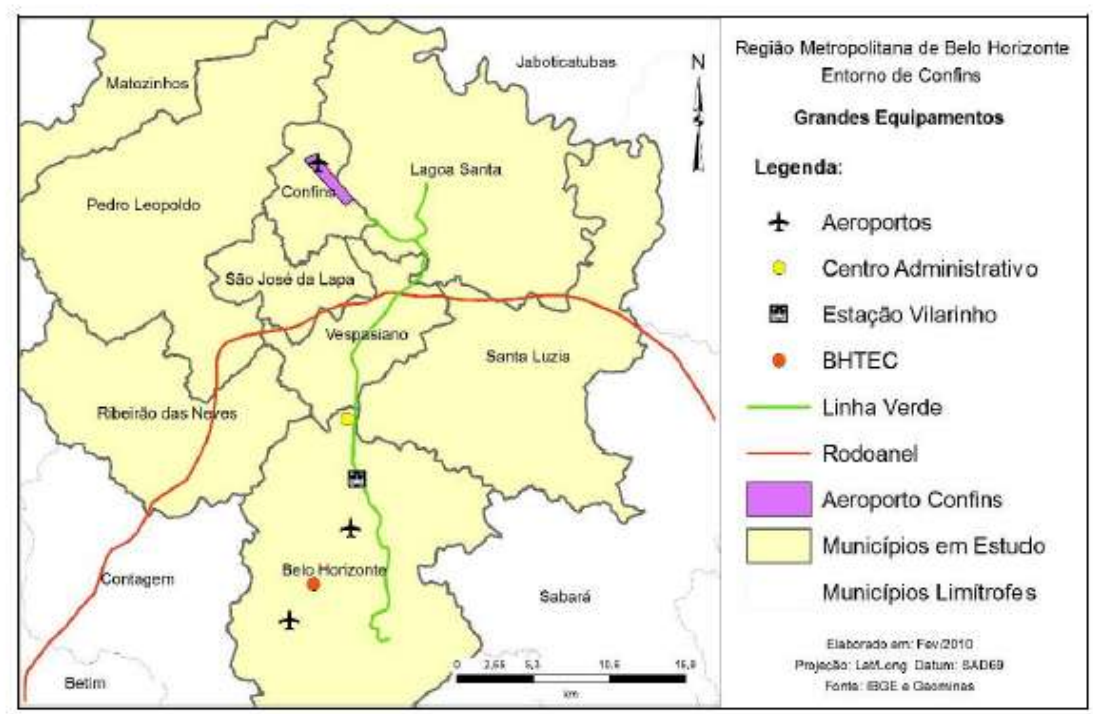

Figura 1: Localização do Eixo Norte e dos Grandes Equipamentos implementados

\section{3 - METODOLOGIA}

Para a elaboração dos mapas em questão, a base sociodemográfica abarcou total ou parcialmente as porções territoriais dos oito municípios inseridos no estudo. A unidade mínima onde as informações foram trabalhadas foi o setor censitário, cuja disponibilização pelo Instituto Brasileiro de Geografia e Estatística (IBGE) passou a incorporar, a partir do ano 2000, um maior número de variáveis em nível de setor que compreende as características dos domicílios, dos seus responsáveis e residentes. Para obterinformações nessa escala de trabalho e montar um banco de dados, foi preciso processar os microdados do Censo Demográfico a partir do software SPSS e agrupar os bancos às bases cartográficas.

Entretanto, atendendo à proposta da Pesquisa, as análises de previsibilidade da condição de diferentes variáveis, demandaram a inserção de métodos que englobam procedimentos matemáticos e 
operações com as unidades espaciais utilizadas.Nesse sentido, as projeções populacionais contribuem para o delineamento de políticaspúblicas e para a própria vida socioeconômica de diferentes sedes municipais.

Contudo, projetar populações de pequenas áreas envolve inúmeros problemas, como as dificuldades de detalhamento das áreas, o que compromete o uso de metodologias tradicionais. Apesar da escassez de literatura adequada ao tema, há, porém, alguns estudos que procuram aprimorar o assunto. Entre esses, destacam-se os de WALDWOGEL (1998), de FREIRE e ASSUNÇÃO (2000), de ASSUNÇÃO (2000), deJANNUZI (2002), FIGOLI et al. (2003) e deCARVALHO E GARCIA (2004). Para a Pesquisa, a metodologia empregada foi a proposta por esses últimos autores citados, quando o Método da Participação no Crescimento mostrou-se eficaz na estimação da população total e a técnica das Relações de Coorte, prestou-se para a estimativa da estrutura etária.

Todavia, como o objetivo deste paper é focar apenas a produção do Cartograma e suas vantagens atribuídas, não serão aprofundados tais métodos não-triviais.

Os mapas foram trabalhados na escala aproximada de 1:200.000, com a projeção universal transversa de Mercator, Datum Horizontal South American no fuso 23 Sul. O ambiente de confecção e layout se deu no software ArcGiS 9.1 de maneira padronizada, o que possibilitou uma apresentação visual em série de igual impressão e qualidade, facilitando as análises. Estas descrições foram realizadas em equipe - 3 integrantes - utilizando-se de compreensão auditiva e gravação das discussões dos aspectos relevantes em cada representação cartográfica. Posteriormente, os áudios foram transcritos e transformados em texto fluido, revisados e, parte dele, publicado num documento relatorial da Pesquisa multidisciplinar.

\section{4 - ANÁLISE DE MAPAS-DESTAQUE}

Como o Cartograma produzido contempla uma gama de variáveis e as informações estão distribuídas em 11 mapas centrais, apresentar-se-á aqui apenas três dessas cartas e suas respectivas análises, que dão base tanto a um melhor entendimento da situação da região, quanto do teor descritivo realizado nas considerações de cada um. A fonte das informações (IBGE, 2000) contempla o manuseio de dados para o ano 2000.

\subsection{Densidade Populacional}

O mapa de densidades, estrito senso, acompanha o anterior, mas explicita gradientes de densidades urbanas referidos à população residente nos setores censitários em 2000. A classe de 100 ou mais habitantes por hectare, por exemplo, demarca a metade superior de maiores densidade na região. Embora predominem setores censitários com menos de 100 pessoas por hectare, há, vários subespaços 
do Vetor Norte relativamente densos, notadamente nos trechos que evoluem para o norte noroeste, ao longo da Linha Verde, como as manchas relativas às áreas centrais dos municípios de Vespasiano e Lagoa Santa.

É evidente que são muitos os setores de alta densidade no município de Belo Horizonte, sobretudo os das classes 200 a 400 e acima de 400 pessoas por hectare. Mas, nas confluências dos limites de Belo Horizonte com os municípios de Ribeirão das Neves, Santa Luzia e parte do município de Vespasiano ocorrem também algumas manchas de alta densidade. São áreas importantes porque nelas os setores censitários já se mostram relativamente pequenos e é visível a presença de diferentes tipos de infraestrutura urbana (como se verá mais a frente), em núcleos dotados de alta centralidade.

No que diz respeito aos espaços próximosao futuro Rodoanel convém ressaltar que realmentesão muito poucas as áreas com densidade elevada (acima de 100 habitantes por hectare), mas na divisa dos municípios de Vespasiano e São José da Lapa há pontos de maior densidade, assim como no trecho do Rodoanel próximo a conurbação de Ribeirão das Neves com Belo Horizonte.

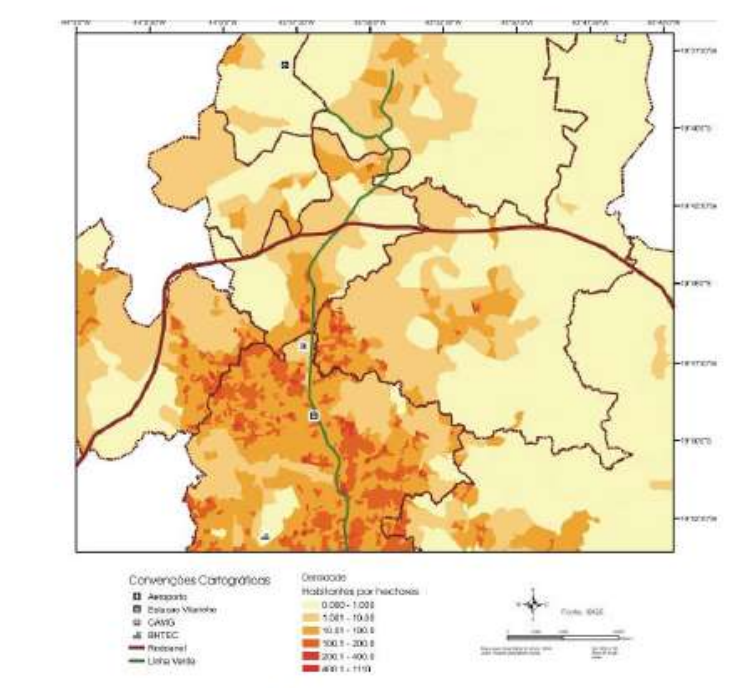

Figura 2: Mapa de Densidade Populacional: habitantes por hectares (2000)

\section{2 - Escolaridade E Renda}

A fim de facilitar a discriminação das principais conclusões, a análise dos níveis de escolaridade dos setores censitários pode ser feita em três grandes grupos. Esses grupos formam-se pelos: i) setores onde a população possui escolaridade média inferior a 5,26 anos de estudo (baixa escolaridade); ii) setores onde as pessoas possuem escolaridade média entre 5,27 a 9,43 (escolaridade média) e iii) setores onde a população possui mais de 9,44 anos de estudo (alta escolaridade).

A primeira conclusão aponta Belo Horizonte como o município que exibe a grande maioria de setores censitários com alta escolaridade, principalmente nas proximidades das áreas centrais e nas 
imediações da lagoa da Pampulha. Fora de Belo Horizonte existem poucoslocais que merecem ser ressaltados, dentre os quais uma pequena área em Lagoa Santa e outra em Vespasiano.

A maior parte das outras áreas mapeadas possui baixa escolaridade, inclusive em vários bairros de Belo Horizonte. Afora esses casos, os dados mostram ser numerosos os setores que ostentam níveis médios de escolaridade, notadamente em Belo Horizonte.

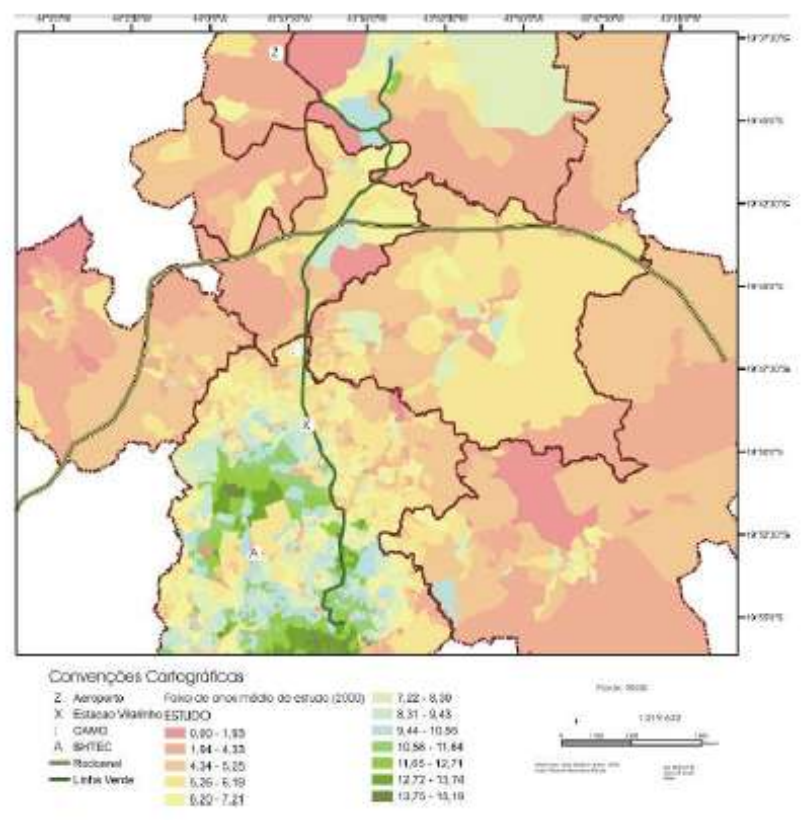

Figura 3: Mapa da faixa de anos médio de estudo (2000)

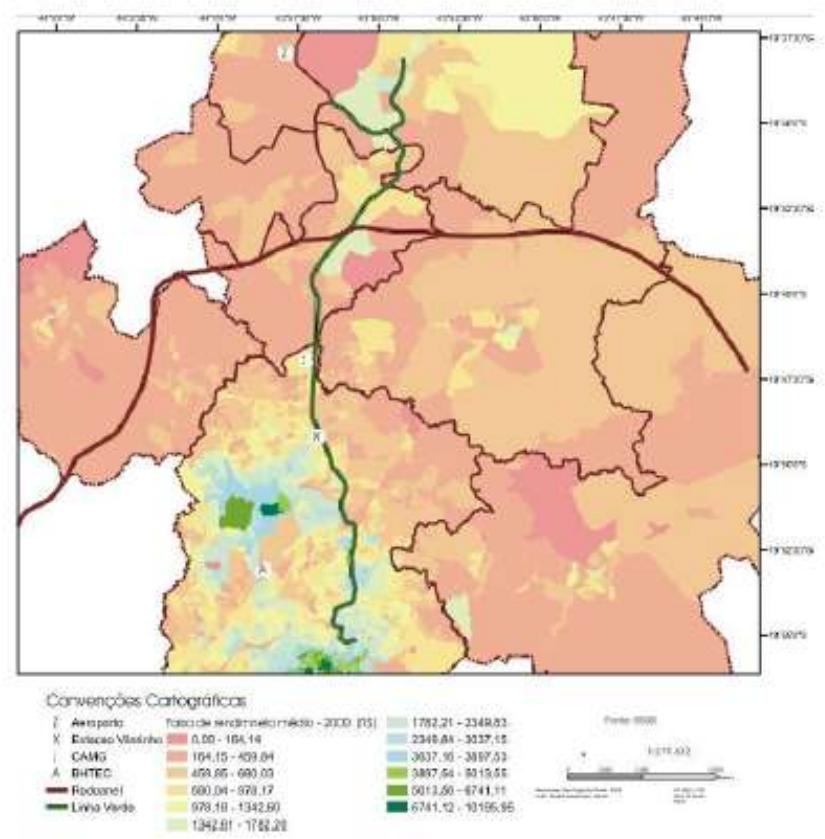

Figura 4: Mapa da faixa de rendimento médio (2000). 
A observação da renda domiciliar indica conclusões semelhantes. Consideradas as oito classes de renda, verifica-se uma concentração de famílias de alta renda nas proximidades da área central e no entorno da lagoa da Pampulha. Fora destas áreas o que predomina são as faixas de média renda (de 680 reais até 2.350 reais por mês), no município de Belo Horizonte e em amplas áreasdo Vetor Norte. São poucos os setores censitários em Belo Horizonte que detém rendas elevadas, no geral predomina a população de baixa renda.

À propósito, vale dizer que a comparaçãodos mapas de renda e de escolaridade, permiteconcluir que a distribuição de renda é claramente mais perversa do que a distribuição de equipamentos associadas a provisão de instrução formal. Há mais setores onde a população tem acesso a níveis de escolaridade médios do que no caso da renda.

\section{3 - Situação Domiciliar Por Setor Censitário}

A situação domiciliar dos residentes no Vetor Norte é predominantemente urbana, mas com um teor rural bastante expressivo. A ocorrência urbana fica bem evidente, particularmente quando se focaliza a categoria área urbanizada de cidade ou vila conforme o mapa de Situação por setor censitário, apresentado à frente. Além dessa categoria, é importante destacar os espaços que se inserem nos chamados aglomerados subnormais, por sua importância do ponto de vista sócio urbanístico. Possuem presença marcante na região embora quase nunca ocupem manchas urbanas contínuas muito extensas.

Há uma profusão de assentamentos especiais do tipo favela distribuídos por boa parte da porção norte do município de Belo Horizonte, assim como a oeste, onde surgem vários desses assentamentos à medida em que se avance para norte ao longo da Linha Verde. No município de Ribeirão das Neves, em área conurbada bem próxima de Belo Horizonte, também comparecem algumas áreas favelizadas. Tais aglomerados também se distribuem pelos municípios de Vespasiano, Santa Luzia e em parte da área limítrofe entre Belo Horizonte e Sabará. Trata-se de espaços relevantes porque são diferenciados edevem acumular especificidades passíveis de serem examinadas em estudos mais detalhados.

Outro tipo de ocupação urbana digna de nota são as chamadas áreas urbanizadas do tipo isoladas. As principais encontram-se a norte, no município de Vespasiano e a leste, no município de Sabará. A análise dessas áreas suscita a indagação sobre porque se encontram isoladas e se mantém expressivas nessa porção do espaço metropolitano. Em alguns casos isso se explica pelo surgimento de condomínios fechados, em outros caberia investigar melhor.

Fica evidente que no interior do município de Belo Horizonte, as áreas não urbanizadas são residuais e muito pouco relevantes em termos de superfície líquida, à exceção da "região" do Isidoro, 
sem dúvida a principal área ainda não urbanizada de Belo Horizonte. Já no município de Ribeirão das Neves essas áreas são bem mais explícitas (ver mapa), tanto quanto em Santa Luzia e municípios circunvizinhos. São áreas isoladas que gozam de uma situação institucional específica, porquanto internalizam expectativas de expansão da ocupação urbana.

No tocante às áreas de características mais rurais duas delas merecem atenção. Primeiramente a mais clássica, a zona rural, que inclusive exclui os chamados aglomerados rurais. Há um número relativamente grande de áreas a oeste dos municípios de Santa Luzia e Sabará cujos setores censitários são predominantemente rurais. Os municípios de Vespasiano, Confins, Lagoa Santa e São José da Lapa também apresentam manchas consideráveis não só nessa categoria, mas também possuem diversos setores classificados em outra: oaglomerado rural isolado.

A visualização da localização das áreas rurais evoca alguma reflexão sobre onde as pressões imobiliárias podem estar ocorrendo. É de se esperar que tais pressões se fazem presente nasproximidades dos interstícios conurbados de Belo Horizonte com os municípios vizinhos. Onde estão as evidências mais nítidas de conurbação no Vetor Norte? É fato que a urbanização de Belo Horizonte está efetivamente espraiada em municípios de seu entorno, embora em vários deles as áreas rurais ainda compareçam em proporções muito significativas.

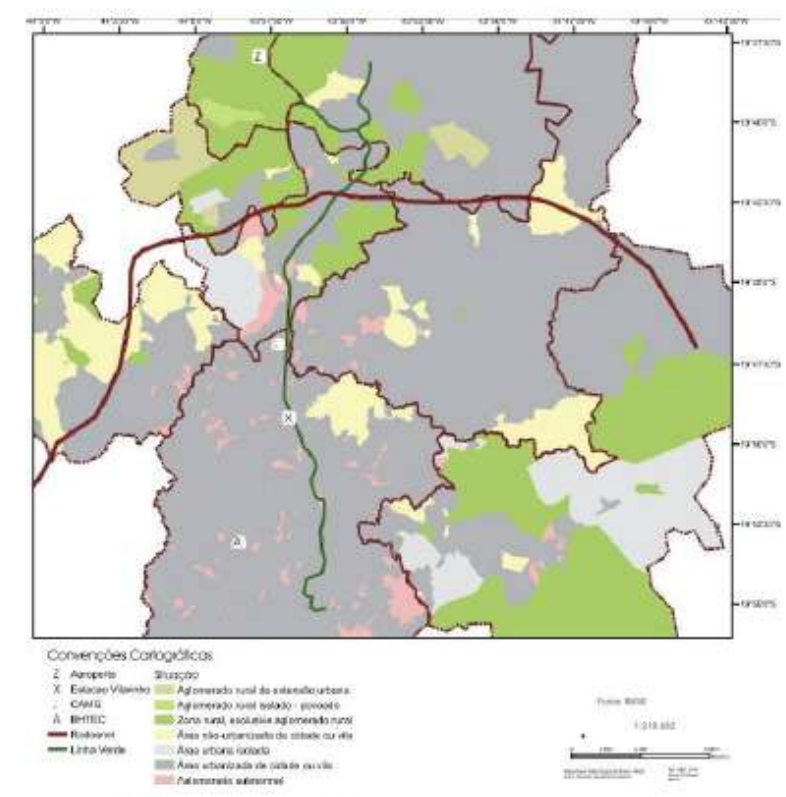

Figura 5: Mapa de Situação domiciliar (2000)

Em direção a norte, uma extensa área urbanizada de contato abrange boa parte do município de Santa Luzia, evoluindo até atingir o município de Sabará. Há, portanto, uma forteconurbação a norte que evolui em direção a nordeste e prossegue aglutinando parte do município de Santa Luzia. A oeste, 
no entorno da Linha Verde há outra série de conexões envolvendo diversas vias urbanas articuladas com o município de Ribeirão das Neves, as quais chegam a fazer contato com uma área urbana isolada do município de Vespasiano. Esses espaços também possuem conexões com o município de São José da Lapa,apesar da existência de áreas rurais nos interstícios próximos.

Considerando a localização relativa dos grandes equipamentos que estão sendo implantados no Vetor Norte algumas questões devem ser ressaltadas. No que diz respeito à Linha Verde, vale dizer que a grande maioria das áreas de seu entorno são claramente urbanas, embora haja algumas exceções no município de Lagoa Santa e em Confins. Quanto ao Anel Viário de Contorno Norte (Rodoanel), cabe observar que, embora também atravesse uma série de áreas que possuem o estatuto de áreas urbanizadas, em seu entorno existem vários setores censitários localizados em áreas rurais, principalmente em Vespasiano. Outros espaços que estão na condição de áreas não urbanizadas de cidade ou vila, comparecemespecialmente no município de Ribeirão das Neves.De toda a forma, o Rodoanel será implantado em área com pouca urbanização consolidada.

Já o Centro Administrativo de Minas Gerais está envolvido em espaços fortementeurbanizados. O Empreendimento está sendo lançado em área urbanizada de cidade ou vila, não obstante a existência de espaços vazios significativos, nos quais, inclusive, se prevê a institucionalização de zonas de proteção e preservação ambiental (sob a forma de parque urbano), informação ainda não disponível no mapa aqui examinado.

\section{5 - CONSIDERAÇÕES}

A elaboração de um conjunto de cartas temáticas se mostrou eficiente à proposta de formulação de alternativas e debate das problemáticas e fatores positivos conjunturais do Eixo Norte da RMBH. Uma descrição minuciosa individual e a síntese dos aspectos relevantes de cada distribuição espacial das variáveis, possibilitou fazer apontamentos de cunho socioeconômicocapazes de nortear o direcionamento das políticas públicas a serem implementadas em prol do bem estar da sociedade.

Um dos diagnósticos de relevância e é a necessidade de melhoramento das áreas dos municípios metropolitanos periféricos em termos de regularização da terra, incentivos à educação e criação de novas oportunidades de trabalho fora de Belo Horizonte. Tais alternativas poderiam, em longo prazo, propiciar um ganho favorável nos índices de habitação, renda e escolaridade, além de aliviar o sistema de fluxos pendulares em boa parte da Região Metropolitana de Belo Horizonte.

É importante ressaltar o papel da Cartografia aliado ao conhecimento e competências no âmbito Demográfico, desde a capacidade técnica empregada para a elaboração / manuseio do banco de dados e criação dos mapas, até a atenção despendida nas análises. 


\section{6 - BIBLIOGRAFIA}

ASSUNÇÃO, R. M. Método Bayesiano de Relação de Coortes para Projeções de PequenasÁreas. In: Encontro Nacional de EstudosPopulacionais, 13, 2002, Ouro Preto, MG. BeloHorizonte: ABEP, 2002.

CARVAlHO, J. A. M., GARCIA, R. A. Projeçãoanual da população das unidades de planejamento(up's) do município de Belo Horizonte, segundosexo e idade simples, entre 2000 e 2010. In:

LEMOS, M. B., DINIZ, C. C., CARVALHO, J. A.M., SANTOS, F. Belo Horizonte no Século XXI. Belo Horizonte: Cedeplar/UFMG, $2004 . \quad$ Disponívelem: www.cedeplar.ufmg.br/pesquisas/projeto_pbh

FÍGOLI, Moema Gonçalves Bueno, WONG,Laura Rodríguez, SAWYER, Diana Oya,CARVALHO, José Magno de. Proyecciónmultirregional: aplicaciónen Brasil y sus unidadesfederativas (2000-2020). Notas de Población 76.2003, p. 7-45

FREIRE, F. H. M. A; ASSUNÇÃO, R. M.Projeção Populacional com EstimadoresBayesianos Espaciais. In: Encontro Nacional deEstudos Populacionais, 13, 2002, Ouro Preto, MG.Violência, estado e qualidade de vida da populaçãoBrasileira. Belo Horizonte: ABEP, 2002.

IBGE, Censo Demográfico de 1970, 1980, 1991 e2000. Rio de Janeiro: IBGE, 2000.

JANNUZZI, P. M. Projeções Populacionais paraPequeníssimas Áreas: método e resultados para osdistritos da cidade de São Paulo em 2010. In:Encontro Nacional de Estudos Populacionais, 13,2002, Ouro Preto, MG. Violência, estado equalidade de vida da população Brasileira. BeloHorizonte: ABEP, 2002. 